\title{
Using Sound in Multi-Touch Interfaces to Change Materiality and Touch Behavior
}

\author{
Ana Tajadura-Jiménez ${ }^{1}$, Bin Liu ${ }^{1}$, Nadia Bianchi-Berthouze ${ }^{1}$, Frédéric Bevilacqua ${ }^{2}$ \\ ${ }^{1}$ UCL Interaction Centre \\ UCL, London, UK \\ [a.tajadura@,b.liu.12@alumni., \\ nadia.berthouze@]ucl.ac.uk \\ ${ }^{2}$ STMS Lab \\ IRCAM-CNRS-UPMC \\ Paris, France \\ Frederic.Bevilacqua@ircam.fr
}

\begin{abstract}
Current development in multimodal interfaces allows us to interact with digitally represented objects. Sadly, these representations are often poor due to technical limitations in representing some of the sensorial properties. Here we explore the possibility of overcoming these limitations by exploiting multisensory integration processes and propose a sound-based interaction technique to alter the perceived materiality of a surface being touched and to shape users' touch behavior. The latter can be seen both as a cue of, and as a means to reinforce, the altered perception. We designed a prototype that dynamically alters the texture-related sound feedback based on touch behavior, as in natural surface touch interactions. A user study showed that the frequency of the sound feedback alters texture perception (coldness and material type) and touch behavior (velocity and pressure). We conclude by discussing lessons learnt from this work in terms of HCI applications and questions opened by this research.
\end{abstract}

\section{Author Keywords}

Auditory texture perception; multimodal interfaces; sonification; surface interaction; touch interface.

\section{ACM Classification Keywords}

H.5.2. User Interfaces: Auditory (non-speech) feedback; interaction styles; input devices and strategies; haptic i/o.

\section{INTRODUCTION}

With the current development in multimodal interfaces, we increasingly interact with digitally represented objects that we explore through touch. Let's think to devices such as $i$ shoogle [2] to handle textiles digitally, e-paper [11], touchscreen widgets [10], apps for shopping materials online or for handling objects remotely during touch-less surgery [17]. Adding texture information to a digitally represented surface is often challenging but is critical to preserve the naturalness of the touch experience [20].

Permission to make digital or hard copies of part or all of this work for personal or classroom use is granted without fee provided that copies are not made or distributed for profit or commercial advantage and that copies bear this notice and the full citation on the first page. Copyrights for thirdparty components of this work must be honored. For all other uses, contact the Owner/Author.

Copyright is held by the owner/author(s).

NordiCHI '14, Oct 26-30 2014, Helsinki, Finland ACM 978-1-4503-2542-4/14/10.

http://dx.doi.org/10.1145/2639189.2639217
Haptic interfaces can provide texture information to some extent, but are not yet able to provide the full texture experience and are not widely used. Thus, researchers are turning into other modalities that provide realistic perceived textures while being easily integrated into current interfaces. Vision [15] and touch [1] are the most explored but still limited in the texture experience they provide.

Sonification may be used to overcome limitations in representing tactile properties of digital objects, which opens new avenues in the design of interfaces. Sound can highlight either specific texture properties or an overall texture feeling. The former may help the design of clothing or digital textile handling apps, as it may allow an insight on how the textile may feel when touched or worn [2]. In fact, the use of textural sounds is known to result in longer interaction time with a clothing product and willingness to pay more for it [9]. The latter is often sought when designing luxury products (feeling a "expensive texture").

Here we propose and evaluate a sound-based interaction technique to change (1) the perceived materiality of a touched surface and (2) the touch behavior as users touch it. We study changes in touch behavior not only as a measure of changes in perceived materiality but also a target effect as it may strengthen the altered perception and help maintaining it. In fact, proprioceptive feedback from touching interacts with other sensory inputs (sound and touch) and it will work in support of keeping a congruent sensory experience. We can consider a sensorimotor loop by which altering the sensory output from surface touch interactions changes the expectations of how it feels to touch that surface [20]; this impacts on the way of touching it which in return impacts on the sensory output.

We expect that texture-related sound feedback will shape the perceived materiality and touch behavior, since:

(1) the frequency variation of the vibrotactile signals received when touching a surface informs of texture [16];

(2) frequency channels are perceptually linked across audition and touch [23]; thus changes in sound frequency may lead to changes in tactile perception; and

(3) during actions, single coherent percepts arise even when discrepancies between expected and actual sensory outputs occur. If kept within certain limits, these discrepancies are compensated by doing fine motor adjustments [13]. 


\section{Related work: Representing texture with sound}

Texture, the microstructure of an object surface, is an important material quality, perceived multisensorially and multidimensionally [14]. It is informed by haptics, vision and audition, and by roughness, hardness, coarseness and thermal dimensions. Roughness is primary for object recognition [16] - while sound is often thought to have little influence on it, especially if touch is available [14], recent studies have shown that the perceived roughness of a touched surface increases if dynamically augmenting the sound high frequency components $[7,12]$ or if increasing the inter-element spacing of the surface grooves [14].

Recent research has exploited the natural audio-motor link by which motor behavior when touching materials continuously "sculpts" the feedback sound. There are now systems that alter the perceived qualities of natural [18] and virtual haptic surfaces [16] by mapping texture/non-texture sounds to motor behavior (e.g. a force-feedback device modulates sound frequency). Fewer studies have instead investigated if texture sounds can alter motor behavior during surface interaction. Hearing on movement onset the sound that will be produced by touching the material of the object, speeds up the reaching-to-grasp movement [5]. Hearing footsteps sounds on different ground surfaces seems to influence participants' walking style [4], though these results did not reach significance, perhaps due to participants being over-conscious about their movement, as they were asked to walk in a specific emotion-related style.

We advance this research by aiming to alter both surface material perception and touch behavior by dynamically changing texture-related sound cues while people are touching the surface. We build on the study by Bresin et al. [4], but focusing in touch interactions rather than full-body movement, and without requesting any specific touch style.

\section{TEXTURE SONIFICATION: SYSTEM OVERVIEW}

Overall, our software allows for tactile-to-audio synthesis in which motor behavior sculpts the sound feedback as in natural surface interactions. Here, a piezo sensor picks up the mechanical vibrations produced when touching a surface with a finger (Figure 1); piezo signal energy thus depends on the finger pressure. Sonification is achieved by real-time ${ }^{1}$ sound analysis/granular synthesis, in which sound samples (grains) from a pre-selected/analyzed sound file that match the piezo signal energy are played back. The synthesis system uses the Max6 MuBu library ${ }^{2}$, that allows designing granular synthesis while controlling specific sound characteristics of each sound grain [19]. Notably, we look at how further changes in sound feedback (i.e. sound timbre) that are not matched to motor behavior affect the subsequent behavior and perceived material.

\footnotetext{
${ }^{1}$ The mean system latency (software + hardware) is $12 \mathrm{~ms}$.

2 Available at http://forumnet.ircam.fr. Software, questionnaire and all study results are available at www.smallinks.com/2R33
}

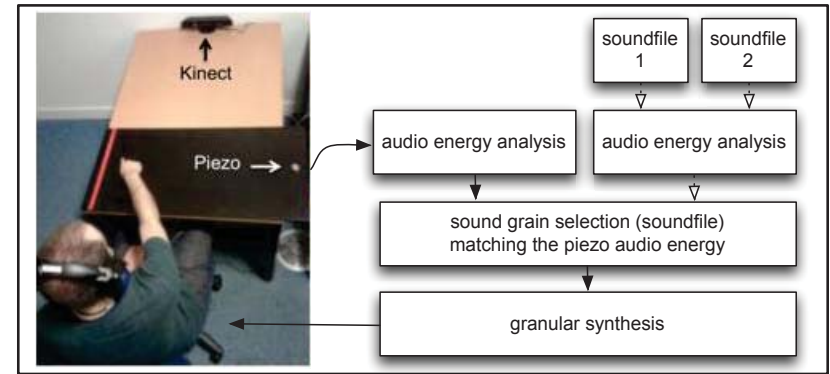

Figure 1. Experimental setup and sound synthesis model. Closed headphones with high ambient noise attenuation (Sennheiser HDA300) masked the real interaction sounds. Headphones and piezo (Schaller Oyster723) connected to a RME FirefaceUC soundcard. Finger velocity was tracked with Kinect and specifically designed software (OpenNI SDK).

\section{USER EXPERIMENT: METHOD}

Materials: Two sounds ${ }^{3}, \mathrm{~S} 1$ and $\mathrm{S} 2$, were chosen for representing a "grainy" surface (rice grains falling into a metal bowl) and a "smooth" surface (gong after stroking a steel bell). Note that the granular synthesis alters the sound so that its cause (i.e. rice, gong) cannot be identified any longer. This sound choice allowed exploring two different ways of altering perceived texture roughness from sound properties (i.e. low/high frequency components and noisiness [7,12,14]). Using granular synthesis we can control, first, high frequency components of any grain sound, which results in modifying the mean high frequency content of the synthesized sound. Second, we can also control the "grainy/roughness" quality of the resulting sound by varying the mean sound grain size, the sound grain density and the grain superposition. These sound transformations are perceived as altering the inter-element spacing of a rough surface, and we expected them to have a large impact on touch behavior (velocity, pressure) and perceived texture properties (roughness and two interrelated dimensions, hardness and thermal qualities [14,22]).

To better test our system and increase the statistical power we used only 2 sounds, with 3 different frequency levels, low (LF), medium (MF) and high frequency (HF). We used Max6 ircamdescriptors $^{2}$ to characterize the sounds. Table 1 shows that LF, MF and HF versions differ by the spectral content of the sound (see the spectral centroid). S1 and S2 differ in their level of "noisiness" (ratio noise energy-total energy). S1 is significantly noisier, while S2 contains more harmonic contents (from the Gong resonance) and retains the "metallic" characteristics of the original sound.

The actual surface being touched was a wooden surface (i.e. a different material than the one used in the sounds). This surface will impact on behavior and assessment of surface qualities, but having the same surface across all conditions allows assessing the sound impact on these measures.

Procedure: 28 paid participants (age $=19-35,9$ male, normal

${ }^{3}$ Freesound database, sounds 59888 and $160513(44.1 \mathrm{kHz})$. 


\begin{tabular}{|c|c|c|c|c|c|c|c|c|c|}
\hline Sound & Centroid & Noisiness & Rough & Hard & Cold & Paper & Sandpaper & Pressure & Velocity \\
\hline S1 LF & $1271(826)$ & $.95(.08)$ & $3(3)$ & $5(2)$ & $4(2)$ & $1(2)$ & $1(2)$ & $2.35(.87)$ & $2.41(.24)$ \\
\hline S1 MF & $1992(941)$ & $.93(.90)$ & $3(3)$ & $5(1)$ & $4(2)$ & $1(1)$ & $1(1)$ & $2.68(.81)$ & $2.44(.27)$ \\
\hline S1 HF & $4029(793)$ & $.83(.12)$ & $3.5(2)$ & $5.5(3)$ & $4(1)$ & $1(2)$ & $1(1)$ & $2.61(.80)$ & $2.40(.28)$ \\
\hline S2 LF & $688(169)$ & $.43(.14)$ & $3(2)$ & $6(2)$ & $5(1)$ & $1(1)$ & $1(1)$ & $2.58(.91)$ & $2.43(.25)$ \\
\hline S2 MF & $1234(193)$ & $.69(.09)$ & $3(3)$ & $5(1)$ & $5(1)$ & $1(2)$ & $1(1)$ & $2.59(.67)$ & $2.40(.26)$ \\
\hline S2 HF & $2203(322)$ & $.71(.07)$ & $4(2)$ & $5(1)$ & $5(2)$ & $2(2)$ & $2(4)$ & $2.60(.75)$ & $2.45(.25)$ \\
\hline
\end{tabular}

Table 1. Mean(SD) for sound descriptors (spectral centroid in $\mathrm{Hz}$ and noisiness), median(IQR) for questionnaire data (7-point scales) and mean(SD) for finger pressure and velocity (which were log-transformed). Gray background marks significant effects.

hearing and touch) naïve as to the study aim took part. They were blindfolded to increase concentration in audio-tactile cues, and to avoid visual texture cues. They completed six experimental blocks differing in the sound feedback (S1 and S2, in their LF, MF and HF versions); their order was randomized. In each block participants kept their right finger in contact with the surface and performed a 1-minute free tactile surface exploration (SE), followed by a distance task (DT). In DT for 5 times, when signaled by a beep, they displaced the finger rightwards a distance of about $50 \mathrm{~cm}$. This distance, starting position, direction of movement number of repetitions, posture and finger used were fixed in order to compare touch behavior across conditions. Blocks were preceded by a silent tactile exploration, and followed by a questionnaire ${ }^{2}$ that assessed (1) the perceived surface qualities of roughness, hardness and coldness [22]; and (2) the likeness the surface was paper, metal, textile, sandpaper, glass, wood, foam and cardboard (based on [3]).

\section{RESULTS}

Table 1 displays the mean effects of sound frequency on (1) texture perception (roughness, hardness, coldness and type of material), and (2) touch behavior: pressure applied in SE (mean square amplitude of piezo signal energy during 5-55 s) and finger velocity in DT $[6,8]$ (mean from start to end, over 5 repetitions). Note that while finger pressure is used both to sculpt the sound feedback and as an index of behavior changes (auditory-motor loop), velocity is only used as index of behavior changes. Parametric (ANOVA, $t$ tests), and non-parametric (Friedman, Wilcoxon) tests were used with normal/non-normal data. Holm-Bonferroni corrected for multiple comparisons, Shaphiro-Wilk tested normality. We report significant effects for $\mathrm{S} 1$ and $\mathrm{S} 2^{2}$.

\section{Perceived texture properties}

Frequency did not significantly alter roughness (S1 HF vs. S1 LF: $z=-1.84, p=0.065$; S2 HF vs. S2 LF: $z=-1.63 ; p=$ $0.103)$, but it affected other interrelated texture dimensions: -In $\mathbf{S} 1$ coldness was affected $(\chi 2(2)=7.83, \mathrm{p}=0.02)$, with the texture being perceived as warmer in S1 LF than in S1 MF $(\mathrm{z}=-2.21, \mathrm{p}=0.027)$ and $\mathrm{S} 1 \mathrm{HF}(\mathrm{z}=-2.58, \mathrm{p}=0.010)$.

-In $\mathbf{S 2}$ the texture was perceived as more paper$(\chi 2(2)=11.31, p=0.004)$ and sandpaper-like $(\chi 2(2)=6.45$, $\mathrm{p}=0.04$ ) in S2 HF than S2 LF (paper: $\mathrm{z}=-3.00, \mathrm{p}=0.003$; sandpaper: $\mathrm{z}=-2.47, \mathrm{p}=0.014$ ) and $\mathrm{S} 2 \mathrm{MF}$ (paper: $\mathrm{z}=-2.26$, $\mathrm{p}=0.024$; sandpaper: $\mathrm{z}=-2.33, \mathrm{p}=0.02$ ).

\section{Touch behavior}

-In S1 finger pressure was affected $(\mathrm{F}(2,52)=5.33$, $\mathrm{p}=0.008)$, with less pressure applied in S1 LF than S1 MF $(\mathrm{t}(26)=-2.63, \mathrm{p}=0.013)$ and $\mathrm{S} 1 \mathrm{HF}(\mathrm{t}(26)=-2.57, \mathrm{p}=0.016)$. -In S2 finger velocity was affected $(\mathrm{F}(2,54)=3.95$, $\mathrm{p}=0.025)$, with slower movements for S2 MF than S2 LF $(\mathrm{t}(27)=-2.07, \mathrm{p}=0.048)$ and $\mathrm{S} 2 \mathrm{HF}(\mathrm{t}(27)=-2.57 ; \mathrm{p}=0.016)$.

\section{DISCUSSION}

We propose a sound-based interaction technique to simulate surface material properties and to shape users' touch behavior when interacting with a multi-touch interface. Touch behavior dynamically controls the texture-related sound feedback. Our evaluation study showed the effectiveness of the technique to induce perceived texture changes across some dimensions. Interestingly, increasing sound frequency resulted in different effects depending on the sound type: for S1 the surface was perceived as colder, and for S2 as more "paper-like" (see also [12]). We also show that increasing sound frequency shaped users' touch behavior. Again, we found different effects for S1 (finger pressure applied increased) and S2 (speed was modified).

Our work exploits the multisensory nature of texture perception [14]. While there might be a hierarchy in the weights given to touch and sound cues during texture perception, we here show that sound cues alone can actually alter the perceived texture $[7,12]$. Crucially, we suggested a sensorimotor interaction loop. Although our feedback actually introduces audio-tactile discrepancies, the observed changes in touch behavior may have contributed to maintain the texture illusion created by the sound. For S1 pressure changes may match perceived changes in the grainy quality of the surface, while for S2 velocity changes may match perceived changes in the paper-like quality. Coherent sensory percepts often arise even when sensory discrepancies exist; if kept under certain threshold, these discrepancies are compensated by fine adjustments in sensory expectations and motor behavior during tactile exploration [13]. Hence adjustments in touch behavior are possibly the result of and the mean for building a coherent multimodal percept. Here, as the sound was touch-driven by pressure, the magnitude of audio-tactile discrepancies was limited, ensuring the rise of a coherent percept.

Our study highlights the opportunities offered by a careful selection of sounds coupled with a related motor behavior: 
the effects depended not only on the sound transformation but on the original sound used. First, perceived coldness and pressure were more affected for the noisier S1, representing a "grainier" surface, though this effect may link to a change in expectations about the material [22], which by default felt warmer than the more "metallic" S2 (see MF means). Changes in felt temperature may help the representation of materials in touchscreen apps for building and art materials, and to induce positive user experiences, given the known thermal-emotion associations [21]. Second, perceived material type and velocity were more affected for S2, representing a "smoother" surface. A sound with less detail about the surface texture may possibly shift the focus of attention to the type of material being touched. In the case of e-paper [11], enhancing the feeling of drawing on a particular type of paper may improve the users' experience and change their drawing behavior.

This paper highlights the contribution of sound to texture perception and calls for reconsidering the use of sound as a compelling approach to shape tactile surface interactions. Whilst possibly a multimodal feedback is ideal, we should consider the fact that adding sound feedback to digital surfaces is often easier than adding haptic cues. Future research should address the limitations of this study, by testing various inputs for the dynamic feedback (pressure, velocity, simple trigger), the magnitude of the audio-tactile discrepancies to optimize the observed effects and larger sound sets from various texture categories to understand which sounds shift attention away from the tactile feedback, which may possibly relate to properties of skin receptors.

\section{ACKNOWLEDGMENTS}

ESRC "The Hearing Body project" (ES/K001477/1) and ANR “Legos project” (11 BS02 012).

\section{REFERENCES}

1. Ando, H., Miki, T., Inami, M., Maeda, T., SmartFinger: nail-mounted tactile display. In Proc. SIGGRAPH 2002.

2. Atkinson, D., Orzechowski, P., Petreca, B., et al. Tactile perceptions of digital textiles: A design research approach. In Proc. CHI 2013, ACM Press (2013).

3. Bergmann Tiest, W.M., Kappers, A.M.L. Analysis of haptic perception of materials by multidimensional scaling and physical measurements of roughness and compressibility. Acta Psychol, 121, 1 (2006), 1-20.

4. Bresin, R., de Witt, A., Papetti, S., Civolani, M., Fontana, F. Expressive sonification of footstep sounds. In Proc ISon 2010.

5. Castiello, U., Giordano, B.L., Begliomini, C., Ansuini, C., Grassi, M. When ears drive hands: the influence of contact sound on reaching to grasp. PLoS ONE,5(2010).

6. Gao, Y., Bianchi-Berthouze, N., Meng, H. What does touch tell us about emotions in touchscreen-based gameplay? In Proc. TOCHI 2012, ACM Press (2012).
7. Guest, S., Catmur, C., Lloyd, D., Spence, C. Audiotactile interactions in roughness perception. Exp Brain Res, 146, 2 (2002), 161-171.

8. Hertenstein, M.J., Holmes, R., McCullough, M., Keltner, D. The communication of emotion via touch. Emotion, 9, 4 (2009), 566-573.

9. Ho, C., Jones, R., King, S., Murray, L., Spence, C. Multisensory augmented reality in the context of a retail clothing application. In K. Bronner, R. Hirt, \& C. Ringe (Eds.), Audio Branding Academy Yearbook 2012/2013, 167-174. Germany: Nomos Publishers (2013).

10. Hoggan, E., Kaaresjoa, T., Laitinen, P., Brewster, S.A. Crossmodal congruence: the look, feel and sound of touchscreen widgets. In Proc. ICMI 2008, ACM Press (2008), 157-164.

11. Holman, D. et al., PaperWindows: Interaction techniques for digital paper. Proc. CHI 2005. 591-599.

12. Jousmaki, V., Hari, R. Parchment-skin illusion: soundbiased touch. Curr Biol, 8, 6 (1998), R190.

13. Kannape, O.A., Blanke, O. Agency, gain and selfconsciousness. Int J Psychophysiol, 83(2012), 191-199.

14. Klatzky, R.L., Lederman, S.J., Multisensory texture perception. In J. Kaiser, M.J. Naumer (Eds) Multisensory object perception in the primate brain, 211-230. New York: Springer (2010).

15. Lecuyer, A., Burkhardt, J., Etienne, L, Feeling bumps and holes without a haptic inter-face: the perception of pseudo-haptic textures. In Proc. CHI 2004, ACM Press (2004), 239-246.

16. McGee, M.R., Gray, P.D., Brewster, S.A. Mixed feelings: Multimodal perception of virtual roughness. In Proc. Eurohaptics 2002, 47-52.

17. Mentis, H.M., O'Hara, K., Sellen, A., R. Trivedi, Interaction proxemics and image use in neurosurgery. In Proc. CHI 2012, ACM Press (2012), 927-936.

18. Merrill, D., Raffle, H. The sound of touch. In Proc. CHI 2007, ACM Press (2007), 2807-2812.

19. Schnell, N., Röbel, A. et al., MuBu and friends Assembling tools for content based real-time interactive audio processing in Max/MSP. In Proc. ICMC 2009.

20. Tallis, R. The hand: A Philosophical Inquiry into Human Being. Edinburgh University Press, 2003.

21. Williams, L.E., Bargh, J.A., Experiencing physical warmth promotes interpersonal warmth, Science, 322, (2008), 606-607.

22. Wongsriruksa, S., Howesb, P., Conreenc, M., Miodownikb, M. The use of physical property data to predict the touch perception of materials. Materials \& Design, 42 (2012), 238-244.

23. Yau, J.M. et al. Temporal frequency channels are linked across audition and touch. Curr Biol, 19(2009), 561-66. 